\title{
An equation of state from a new coordination number model
}

\author{
Min-Lon Yu, Yan-Ping Chen * \\ Department of Chemical Engineering, National Taiwan University Taipei, Taiwan, Republic of China
}

Received 22 August 1994; accepted 4 February 1995

\begin{abstract}
A new coordination number model for square-well fluid is proposed in this work. This model agrees with the theoretical approximation at low densities. It is also in good agreement with the Monte Carlo computer simulation results for square-well fluids at the high density and the close-packed regions. This new coordination number model is compared with other models from literature and better results are observed from this work, especially at the high density region. An equation of state is derived from this new coordination number model and is written in a perturbed hard sphere form. This equation of state gives satisfactory calculated compressibility factors in comparison with the Monte Carlo computer simulation results of the square-well fluids. This 3-parameter equation of state is employed also for real fluid calculations. Pure component parameters for $\mathrm{n}$-paraffins are regressed and presented. This equation of state yields satisfactory calculation results on vapor pressures and liquid densities.
\end{abstract}

Keywords: Theory; Equation of state; Vapor-liquid equilibria; Coordination number; Nonpolar fluids

\section{Introduction}

Thermodynamic properties of real fluids can be calculated by using assumptions on molecular interactions and their microscopic structures. The square-well (SW) model fluid, having the essential features of repulsive and attractive forces between molecules, is widely employed in theoretical investigations. The microstructures of model fluid is expressed by the coordination numbers, the number of neighboring molecules around a central one. The coordination number depends on the molecular potential function and the pair distribution function. Investigations on the coordination number model using the molecular simulation methods have been presented in literature (eg. Hu et al., 1984; Lee and Sandler, 1987; Lee and Chao, 1987). A thermodynamic equation of state (EOS) can then be developed using the coordination number model and the generalized van der Waals partition function (Sandler, 1985). At a low density limit, the coordination number is written according to the

\footnotetext{
${ }^{*}$ Corresponding author.
} 
exact radial distribution function. Coordination number models of SW fluids were proposed by previous investigators by correlating the Monte Carlo (MC) computer simulation data (Lee and Sandler, 1987; Hu et al., 1984; Lee and Chao, 1987). Guo et al. (1990a) proposed a coordination number model which satisfied the theoretical boundaries at low and high densities. In this work, a new expression of the coordination number model for SW fluids is presented. The new coordination number model also meets the theoretical boundaries at the low density and the close-packed limits and it improves the calculation results at high densities. Unlike the complex expressions in some previous coordination number models, an EOS is easily derived from this new model by employing the generalized van der Waals partition function. Comparisons of the thermodynamic properties of pure components and mixtures of the SW fluids are presented in this study. A three-parameter EOS is proposed in this work and is applied in real fluid calculations. We demonstrate that this EOS gives good vapor-liquid equilibrium calculations, especially on the saturated liquid densities for $n$-paraffines.

\section{The coordination number model}

The SW potential function is used in this study

$$
\Gamma(r)=\infty \text { for } r \leq \sigma-\epsilon \text { for } \sigma<r \leq \lambda \sigma 0 \text { for } r>\lambda \sigma
$$

where $\epsilon$ is the energy depth, $\sigma$ is a molecular size parameter and $\lambda$ is a characteristic well width parameter in the SW potential. The coordination number for a SW fluid is calculated by

$$
N_{\mathrm{c}}=4 \pi \rho \int_{\sigma}^{\lambda \sigma} g(r) d r
$$

where $g(r)$ is the pair distribution function. Applying the low density limit expression of $g(r)$ (Reed and Gubbins, 1973), the coordination number of a SW fluid at a low density limit is

$$
\left(N_{\mathrm{c}}\right)_{\rho \rightarrow 0}=\frac{4 \pi}{3} \rho \sigma^{3}\left(\lambda^{3}-1\right) \exp \left(\frac{\epsilon}{k T}\right)
$$

At higher densities, the coordination number equation is expressed as a function of the potential energy parameters as well as the density. Previous models for the coordination numbers of SW fluids are listed in Table 1. As it has been discussed by Guo et al. (1990a); Guo et al. (1990b), each of these models has drawbacks in estimating the coordination numbers at low or high density limits, or has difficulties in integration to obtain an EOS. In this study, we express the coordination number of a SW fluid at any density by multiplying Eq. (3) by a correction term, $\Psi$, which is a function of the density and energy parameter

$$
N_{\mathrm{c}, \rho}=N_{\mathrm{c}, \rho \rightarrow 0} \cdot \Psi
$$

Fig. 1 shows a plot of the ratios of the MC computer simulated coordination number to its low density limit for pure SW fluids over a wide density range and with various energy parameters. The plot in Fig. 1 shows a regular trend and a correlation equation is proposed for the correction term $\Psi$ as a function of the reduced density and the energy parameter. The boundary conditions are considered in 
Table 1

Coordination number models in previous researches

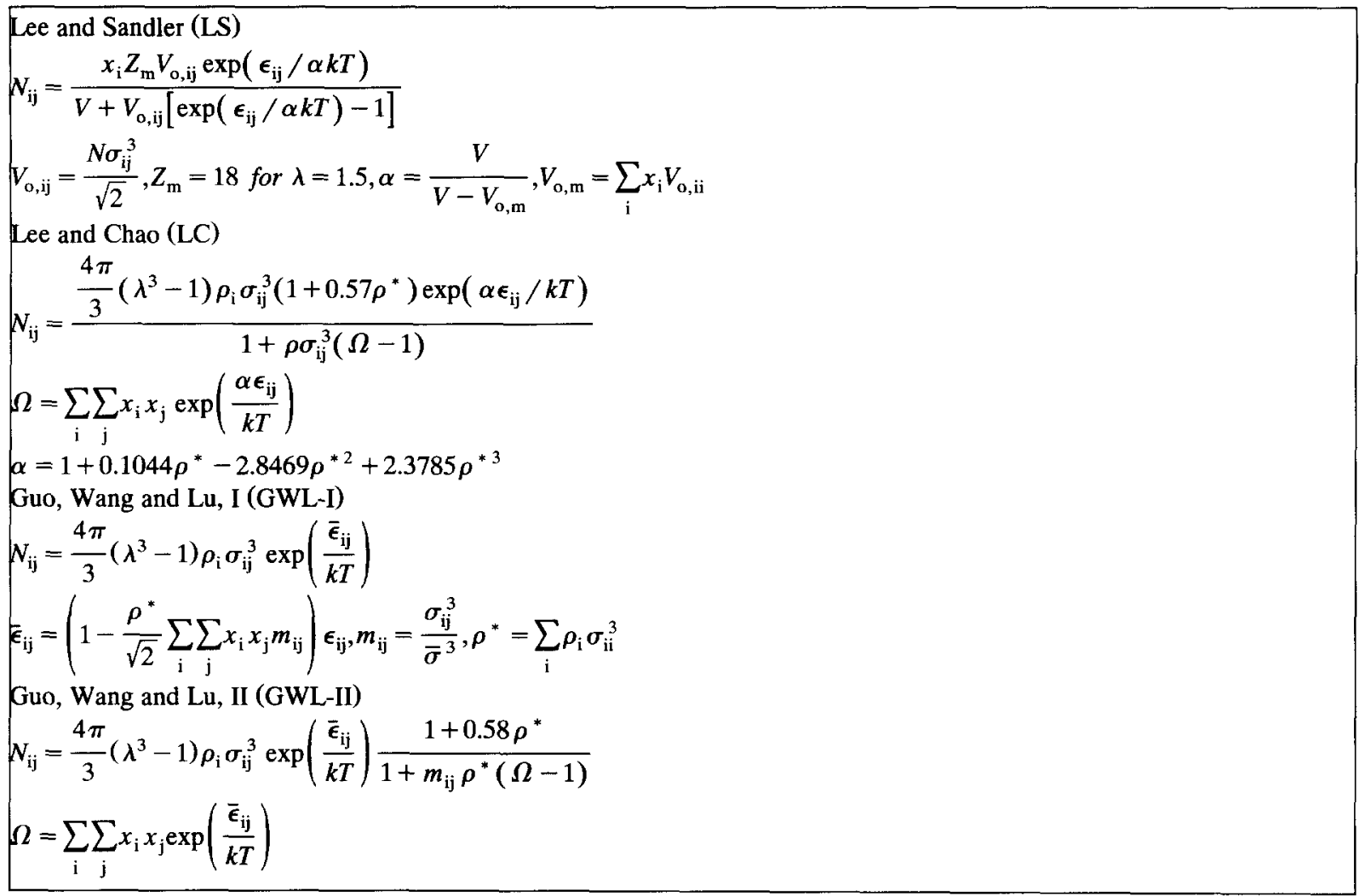

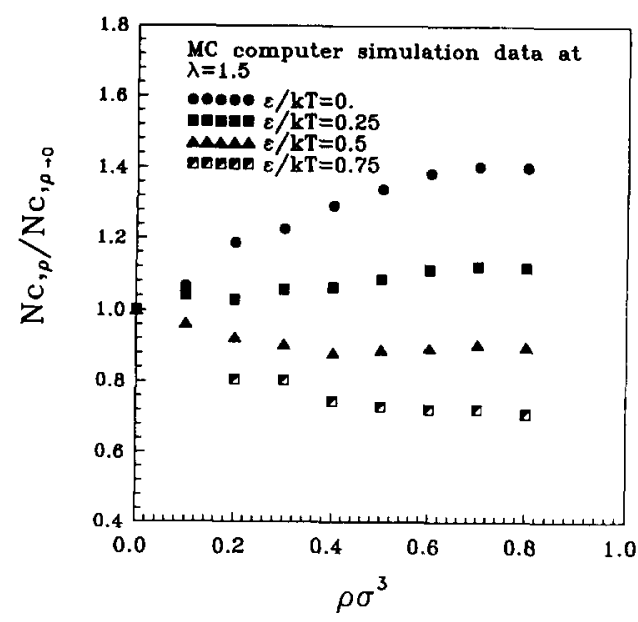

Fig. 1. Plot of the ratio of the coordination numbers of SW fluids to the low density limiting values against the reduced density (MC computer simulation data: Lee et al., 1985). 
Table 2

The comparison of the coordination numbers for pure SW fluids at limiting states by various models

\begin{tabular}{lll}
\hline Model & Low density limit condition & Close-packed condition $(\lambda=1.5)$ \\
\hline Exact & $(4 \pi / 3)\left(\lambda^{3}-1\right) \rho \sigma^{3} \exp (\epsilon / \mathrm{kT})$ & 18 \\
LS & $12.73\left(\lambda^{3}-1\right) \rho \sigma^{3} \exp (\epsilon / \mathrm{kT})$ & 18 \\
LC & $(4 \pi / 3)\left(\lambda^{3}-1\right) \rho \sigma^{3} \exp (\epsilon / \mathrm{kT})$ & $25.41 \exp (2.18 \epsilon / \mathrm{kT}) /(1.41 \exp (2.18 \epsilon / \mathrm{kT})-0.41)$ \\
GWL-I & $(4 \pi / 3)\left(\lambda^{3}-1\right) \rho \sigma^{3} \exp (\epsilon / \mathrm{kT})$ & 14.07 \\
GWL-II & $(4 \pi / 3)\left(\lambda^{3}-1\right) \rho \sigma^{3} \exp (\epsilon / \mathrm{kT})$ & 25.61 \\
This work & $(4 \pi / 3)\left(\lambda^{3}-1\right) \rho \sigma^{3} \exp (\epsilon / \mathrm{kT})$ & 20.4 \\
\hline
\end{tabular}

the correlation that Eq. (3) is satisfied at a low density limit, and the coordination number becomes independent of the energy parameter at a high density limit. The following expression for the coordination number is then proposed in this study

$$
N_{\mathrm{c}}=\frac{4 \pi}{3} \rho \sigma^{3}\left(\lambda^{3}-1\right) \exp \left(\frac{\epsilon}{k T}\right)[1+(1.45 \exp (-\epsilon / k T)-1)(1-\exp (1-\phi))]
$$

where

$$
\phi=\frac{\sqrt{2}+\rho \sigma^{3}}{\sqrt{2}-\rho \sigma^{3}}
$$

Eq. (5) reduces to the low density limit, Eq. (3), as the reduced density $\rho \sigma^{3}$ and approaches to zero. At the close-packed condition, as $\rho \sigma^{3}$ approaches to $2^{0.5}$, Eq. (5) becomes

$$
\underset{\rho \sigma^{3} \rightarrow \sqrt{2}}{\operatorname{Lim}} N_{c}=\frac{5.8 \pi}{3}\left(\lambda^{3}-1\right) \rho \sigma^{3}
$$

Eq. (7) is reasonable that the properties of a fluid depend solely on the reduced density at the close-packed condition. Using $\lambda=1.5$, Eq. (7) gives the close-packed coordination number of 20.4, which is in better agreement to the exact value of 18 for a pure SW fluid at the close-packed condition than that from most models shown in Table 1. A comparison of the coordination numbers of a SW fluid at the low density limit and the close-packed conditions from various models is shown in Table 2. A constant of 1.45 is used in Eq. (5) which is obtained by fitting this equation with the MC computer simulation data for pure SW fluids. Comparisons of our calculated results with the MC computer simulation data and those from other models are presented in the next section. Eq. (5) is extended to mixtures and the coordination number of a unlike pair of molecules $i$ and $j$ is written as

$$
N_{\mathrm{ij}}=\left[\frac{4 \pi}{3}\left(\lambda^{3}-1\right) x_{\mathrm{i}} \rho \sigma_{\mathrm{ij}}^{3} \exp \left(\frac{\epsilon_{\mathrm{ij}}}{k T}\right)\right]\left\{1+\left[1.45 \exp \left(\frac{-\epsilon_{\mathrm{ij}}}{k T}\right)-1\right]\left[1-\exp \left(1-\phi_{\mathrm{ij}}\right)\right]\right\}
$$

where the following expressions for the cross terms are employed in this study

$$
\begin{aligned}
& \sigma_{\mathrm{ij}}=\frac{1}{2}\left(\sigma_{\mathrm{i}}+\sigma_{\mathrm{j}}\right) \\
& \epsilon_{\mathrm{ij}}=\left(\epsilon_{\mathrm{i}} \epsilon_{\mathrm{j}}\right)^{0.5} \\
& \phi_{\mathrm{ij}}=\frac{\sqrt{2}+\rho \sigma_{\mathrm{ij}}^{3}}{\sqrt{2}-\rho \sigma_{\mathrm{ij}}^{3}}
\end{aligned}
$$


Table 3

The comparison of the calculated coordination numbers for pure SW fluids by various models at $\lambda=1.5$

\begin{tabular}{|c|c|c|c|c|c|c|c|c|}
\hline \multirow[t]{2}{*}{$\epsilon / k T$} & \multirow[t]{2}{*}{$\rho \sigma^{3}$ range } & \multirow{2}{*}{$\begin{array}{l}\text { No of } \\
\text { Data Pts }\end{array}$} & \multicolumn{5}{|c|}{ Absolute average deviation $\%^{a}$} & \multirow{2}{*}{$\begin{array}{l}\text { data } \\
\text { sources }\end{array}$} \\
\hline & & & $\overline{\mathrm{LS}}$ & GWL-I & GWL-II & LC & This work & \\
\hline 0 & $0.1-0.8$ & 8 & 7.85 & 21.67 & 3.32 & 3.46 & 2.5 & 1 \\
\hline 0.25 & $0.1-0.8$ & 8 & 4.72 & 14.12 & 1.21 & 1.12 & 0.77 & 1 \\
\hline 0.5 & $0.1-0.8$ & 8 & 3.04 & 6.09 & 1.79 & 2.27 & 3.06 & 1 \\
\hline 0.75 & $0.1-0.8$ & 8 & 3.65 & 6.07 & 3.50 & 3.54 & 6.24 & 1 \\
\hline 0.57 & $0.05-0.8$ & 6 & 2.84 & 5.38 & 2.37 & 2.23 & 2.76 & 2 \\
\hline 0.4 & $0.05-0.8$ & 6 & 4.65 & 8.15 & 1.95 & 2.38 & 2.11 & 2 \\
\hline 0.33 & $0.05-0.8$ & 6 & 5.8 & 9.52 & 1.74 & 2.18 & 1.77 & 2 \\
\hline \multicolumn{3}{|c|}{ Grand average } & 4.68 & 10.44 & 2.30 & 2.48 & 2.81 & \\
\hline
\end{tabular}

Data Sources: 1. Lee et al. (1985); 2. Guo et al. (1990a).

${ }^{\mathrm{a}} \mathrm{AAD}(\%)=\frac{100}{N} \sum_{i}\left(a b s\left(\frac{N c_{\mathrm{i}, \mathrm{MC}}-N c_{\mathrm{i}, \text { cal }}}{N c_{\mathrm{i}, \mathrm{MC}}}\right)\right), N$ : total number of data points.

\section{Comparisons of the new coordination number model}

The new model, shown in Eqs. (5) and (8) for pure fluid and mixtures, respectively, are used to calculate the coordination numbers of the SW fluids with different size and energy parameters. Comparisons of our calculated results with the MC computer simulation data and with those calculated from other models (Lee and Chao, 1987 (LC); Lee and Sandler, 1987 (LS); Guo et al., 1990a (GWL-I, GWL-II); Shen and Lu, 1993 (SL); Tang and Lu, 1994 (TL)) are discussed in this work. Table 3 shows the absolute percentage average deviations of the calculated coordination numbers from the MC computer simulated data by five pure fluid models. It is obvious that our model yields comparably good results as those from the LC and GWL-II models while the LS and GWL-I models show relatively larger deviations. Fig. 2(a) and (b) show the graphical comparisons of our calculated coordination numbers at low and high densities. Generally, our calculated results agree
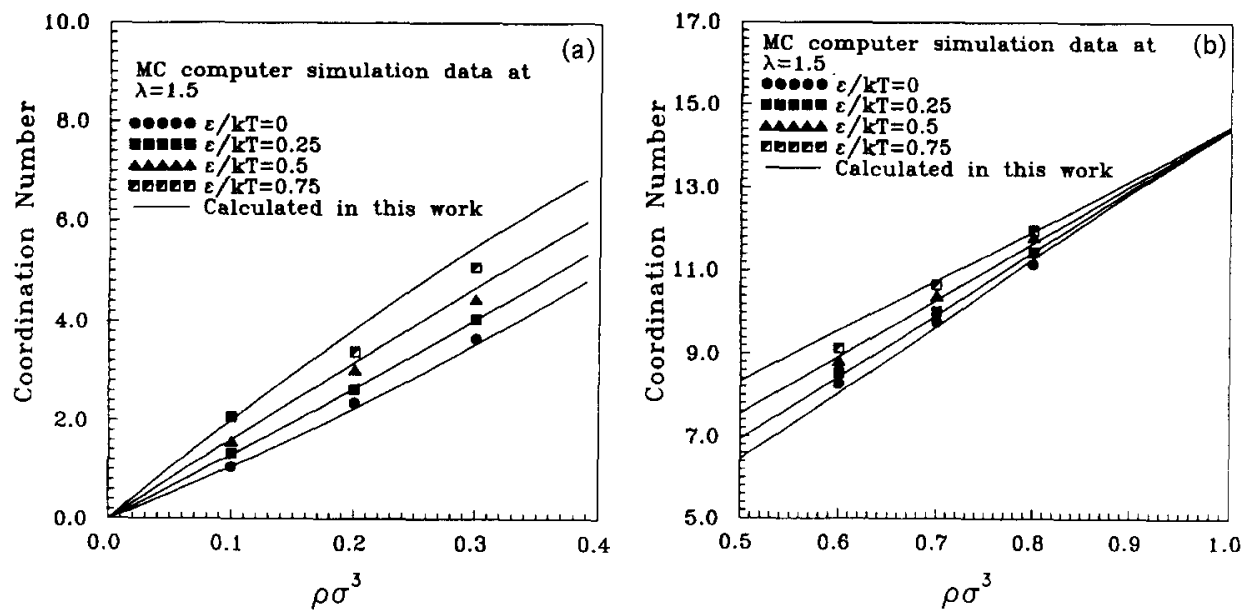

Fig. 2. (a) Coordination numbers of the pure SW fluids calculated in this work at the low density region (MC computer simulation data: Lee et al., 1985); (b) Coordination numbers of the pure SW fluids calculated in this work at the high density region (MC computer simulation data: Lee et al., 1985). 


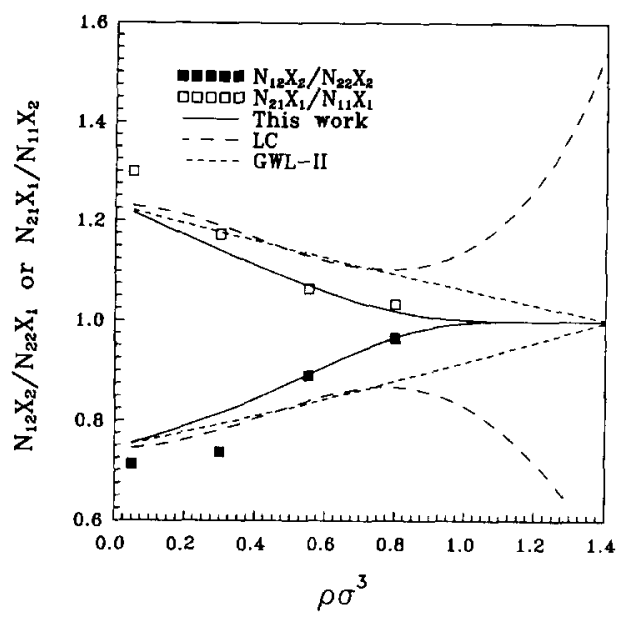

Fig. 3. Comparison of coordination number ratios of SW fluid mixtures calculated by different modles $\left(\epsilon_{11} / k T=0.5\right.$, $\epsilon_{22} / k T=1.0, \epsilon_{12} / k T=0.707, x_{1}=0.204, \sigma_{22} / \sigma_{11}=1$ and $\lambda=1.5 ;$ MC computer simulation data: Lee and Chao, 1987).

well with the MC computer simulation data. Besides the pure fluid results, we have, as shown in Table 4, compared the coordination numbers of binary SW fluid mixtures calculated from different models with the MC computer simulated data. It is observed in Table 4 that the coordination number models of LC and GWL-II give the best results while the models of LS and GWL-I have relatively larger errors. Our proposed model gives slightly larger deviations than those from the models of LC and GWL-II, but shows better results than those from LS and GWL-I. Our model, however, has other advantages. Firstly, our model does not have a complex summation term, $\Omega$, as appeared in the LC and GWL-II models. This makes it easier to derive an EOS through integration of the coordination number equation. Secondly, for an equal-size binary $\mathrm{SW}$ fluid mixture, Lee et al. (1986) has demonstrated that at the close-packed condition, the ratio of $N_{12} x_{2} / N_{22} x_{1}$ or $N_{21} x_{1} / N_{11} x_{2}$ approaches to unity. Fig. 3 shows a plot of these coordination number ratios against the reduced density for an equal-size SW fluid mixture calculated from our model. Fig. 3 also presents the comparisons of our calculated results with those from the LC and GWL-II models and the MC computer simulation data. It is shown that our model agrees well with the MC simulation data and satisfies the boundary condition as the reduced density approaches to the close-packed limit.

\section{Development of an equation of state}

According to the generalized van der Waals theory (Sandler, 1985), the canonical partition function is written as

$$
Q(N, V, T)=\prod_{\mathrm{i}} \frac{1}{N_{\mathrm{i}} !}\left(\frac{q_{\mathrm{r}, \mathrm{v}, \mathrm{e}}}{\Lambda^{3}}\right)^{N_{\mathrm{i}}} V_{\mathrm{f}}^{\mathrm{N}} \exp \left(\frac{-N \Phi}{2 k T}\right)
$$


Table 4

Comparisons of the calculated results of the coordination numbers of binary SW fluids from various models at $\lambda=1.5$

\begin{tabular}{|c|c|c|c|c|c|c|c|}
\hline \multirow[t]{2}{*}{ Case } & & \multicolumn{5}{|c|}{ Absolute average deviation $\%^{a}$} & \multirow{2}{*}{$\begin{array}{l}\text { data } \\
\text { sources }\end{array}$} \\
\hline & & LS & $\mathrm{LC}$ & GWL-I & GWL-II & This work & \\
\hline \multirow[t]{2}{*}{ I } & $N_{\mathrm{ij}}$ & 15.22 & 5.41 & 9.40 & 5.41 & 7.37 & 1 \\
\hline & $N_{\mathrm{ci}}$ & 12.04 & 3.76 & 7.13 & 3.98 & 3.92 & 1 \\
\hline \multirow[t]{2}{*}{ II } & $N_{\mathrm{ij}}$ & 11.64 & 4.04 & 8.76 & 4.65 & 3.96 & 1 \\
\hline & $N_{\mathrm{ci}}$ & 10.81 & 3.49 & 6.97 & 4.12 & 3.58 & 1 \\
\hline \multirow[t]{2}{*}{ III } & $N_{\mathrm{ij}}$ & 10.29 & 6.67 & 10.76 & 7.78 & 7.41 & 1 \\
\hline & $N_{\mathrm{ci}}^{3}$ & 8.07 & 5.31 & 8.54 & 6.55 & 5.18 & 1 \\
\hline \multirow[t]{2}{*}{ IV } & $N_{\mathrm{ij}}$ & 12.18 & 4.05 & 8.57 & 4.03 & 4.28 & 1 \\
\hline & $N_{\mathrm{ci}}$ & 11.41 & 3.21 & 6.66 & 3.18 & 3.43 & 1 \\
\hline \multirow[t]{2}{*}{ V } & $N_{\mathrm{ij}}$ & 12.36 & 3.36 & 4.49 & 3.19 & 4.08 & 2 \\
\hline & $N_{\mathrm{ci}}$ & 11.96 & 2.77 & 3.94 & 2.60 & 3.57 & 2 \\
\hline \multirow[t]{2}{*}{ VI } & $N_{\mathrm{ij}}$ & 9.93 & 7.61 & 9.73 & 8.97 & 8.06 & 2 \\
\hline & $N_{\mathrm{ci}}$ & 8.80 & 7.01 & 9.62 & 8.42 & 7.78 & 2 \\
\hline \multirow[t]{2}{*}{ VII } & $N_{\mathrm{ij}}$ & 11.49 & 2.88 & 6.79 & 2.60 & 3.47 & 3 \\
\hline & $N_{\mathrm{ci}}$ & 11.35 & 2.50 & 5.30 & 2.12 & 2.91 & 3 \\
\hline \multirow[t]{2}{*}{ VIII } & $N_{\mathrm{ij}}$ & 11.62 & 2.71 & 8.99 & 2.04 & 4.09 & 3 \\
\hline & $N_{\mathrm{ci}}$ & 11.44 & 2.33 & 8.15 & 1.69 & 3.15 & 3 \\
\hline
\end{tabular}

Case I: $\epsilon_{11} / k T=0.5, \epsilon_{12} / k T=0.5, \epsilon_{22} / k T=1.0$.

Case II: $\epsilon_{11} / k T=0.5, \epsilon_{12} / k T=0.71, \epsilon_{22} / k T=1.0$.

Case III: $\epsilon_{11} / k T=0.5, \epsilon_{12} / k T=1.0, \epsilon_{22} / k T=1.0$.

Case IV: $\epsilon_{11} / k T=0.33, \epsilon_{12} / k T=0.58, \epsilon_{22} / k T=1.0$.

for Case I-III, $\sigma^{22} / \sigma^{11}=1.0-2.0, x_{1}=0.204-0.796, \rho^{*}=0.05-0.8$.

for Case IV, $\sigma^{22} / \sigma_{11}=1.0-2.0, x_{1}=0.5, \rho^{*}=0.05-0.8$.

Case V: $\epsilon_{11} / k T=0.4, \epsilon_{12} / k T=0.57, \epsilon_{22} / k T=0.8$.

Case VI: $\epsilon_{11} / k T=0.6, \epsilon_{12} / k T=0.85, \epsilon_{22} / k T=1.2$.

for Case V and VI $\sigma_{22} / \sigma_{11}=1.0, x_{1}=0.25-0.75, \rho^{*}=0.1-0.7$.

Case VII: $\epsilon_{11} / k T=\epsilon_{12} / k T=\epsilon_{22} / k T=0.6$.

Case VIII: $\epsilon_{11} / k T=\epsilon_{12} / k T=\epsilon_{22} / k T=0.4$.

for Case VII and VIII $\sigma_{22} / \sigma_{11}=1.2-2.0, x_{1}=0.25-0.75, \rho^{*}=0.1-0.7$.

Data sources: 1. Lee and Chao, 1987; 2. Lee et al., 1986; 3. Lee and Sandler, 1987.

a: $\operatorname{AAD}(\%)=(100 / N) \sum_{k}\left(a b s\left(\frac{N_{\mathrm{ij}, \mathrm{k}, \mathrm{MC}}-N_{\mathrm{ij}, \mathrm{k}, \mathrm{cal}}}{N_{\mathrm{ij}, \mathrm{k}, \mathrm{MC}}}\right)\right), N$ : total number of data points.

Table 5

The comparison of various models in the calculation of the compressibility factors of pure SW fluids at $\lambda=1.5$

\begin{tabular}{|c|c|c|c|c|c|c|c|c|c|}
\hline \multirow{2}{*}{$\begin{array}{l}\text { range of } \\
\epsilon / k T\end{array}$} & \multirow{2}{*}{$\begin{array}{l}\text { range of } \\
\rho \sigma^{3}\end{array}$} & \multirow{2}{*}{$\begin{array}{l}\text { No. of } \\
\text { Data pts. }\end{array}$} & \multicolumn{6}{|c|}{ Absolute average deviation ${ }^{a}$} & \multirow{2}{*}{$\begin{array}{l}\text { data } \\
\text { sources }\end{array}$} \\
\hline & & & $\mathrm{LC}$ & LS & GWL-I & GWL-II & SL & This work & \\
\hline $0.33-0.57$ & $0.05-0.8$ & 18 & 0.17 & 0.22 & 0.39 & 0.17 & 0.12 & 0.08 & 1 \\
\hline $0.09-1.92$ & $0.20-0.88$ & 145 & 0.67 & 0.24 & 0.58 & 0.61 & 0.37 & 0.24 & 2 \\
\hline $0.25-1.25$ & $0.5-0.8$ & 15 & 0.5 & 0.58 & 0.99 & 0.52 & 0.22 & 0.18 & 3 \\
\hline
\end{tabular}

Data Sources: 1. Guo et al. (1990a); 2. Alder et al. (1972); 3. Henderson (1976).

${ }^{\mathrm{a}} \mathrm{AAD}=(1 / N) \sum_{i}\left(a b s\left(Z_{\mathrm{i}, \mathrm{MC}}-Z_{\mathrm{i}, \mathrm{cal}}\right), N\right.$ : total number of data points. 
where the mean potential $\Phi$ is determined using a coordination number model. The EOS is derived from the canonical partition function

$$
P=k T\left(\frac{\partial \ln Q}{\partial V}\right)_{\mathrm{N}, \mathrm{T}}
$$

We apply the Carnahan-Starling equation (Carnahan and Starling, 1969) to determine the free volume term in the canonical partition function, and employ our proposed coordination number model to evaluate the mean potential $\Phi$. The EOS is then expressed as

$$
\begin{aligned}
Z= & \frac{1+\eta+\eta 2-\eta 3}{(1-\eta)^{3}}-4.9742 \sum \sum x_{\mathrm{i}} x_{\mathrm{j}} \rho_{\mathrm{ij}}^{*}\left[\frac{1.45}{T_{\mathrm{ij}}^{*}}-\exp \left(1-\phi_{\mathrm{ij}}\right)\left(\frac{1.45}{T_{\mathrm{ij}}^{*}}+1-\Omega_{\mathrm{ij}}\right)\right. \\
& \left.\times\left(\frac{2 \sqrt{2}-\rho_{\mathrm{ij}}^{*}-\sqrt{2} \phi_{\mathrm{ij}}}{\sqrt{2}-\rho_{\mathrm{ij}}^{*}}\right)\right]
\end{aligned}
$$

where $\rho_{\mathrm{ij}}^{*}=\rho \sigma_{\mathrm{ij}}^{3}, T_{\mathrm{ij}}^{*}=k T / \epsilon_{\mathrm{ij}}, \Omega_{\mathrm{ij}}=\exp \left(\epsilon_{\mathrm{ij}} / k T\right), \eta=\left(\pi \rho_{\mathrm{m}} / 6\right) \sum x_{\mathrm{i}} \sigma_{\mathrm{i}}{ }^{3}$, and the square-well width $\lambda$ is taken as 1.5. Eq. (14) reduces to the pure fluid equation when $x_{\mathrm{i}}$ is equal to unity.

This EOS is employed in real fluid vapor-liquid equilibrium calculations. The criterion of equality of fugacity is used to determine the pure fluid parameters

$$
f_{\mathrm{i}}^{\mathrm{V}}=f_{\mathrm{i}}^{\mathrm{L}}
$$

The fugacity coefficient for a pure fluid is evaluated using the equation of state

$$
\begin{gathered}
\ln \left(f_{\mathrm{i}} / P\right)=4.9742 \rho_{\mathrm{i}} \sigma_{\mathrm{i}}^{3}\left[\exp \left(1-\phi_{\mathrm{i}}\right)\left(1-\exp \left(\epsilon_{\mathrm{i}} / k T\right)+1.45 \epsilon_{\mathrm{i}} / k T\right)-1.45 \epsilon_{\mathrm{i}} / k T\right] \\
-\eta_{\mathrm{i}}\left(3 \eta_{\mathrm{i}}-4\right) /\left(1-\eta_{\mathrm{i}}\right)^{2}-\ln Z_{\mathrm{i}}+\left(Z_{\mathrm{i}}-1\right)
\end{gathered}
$$

\section{Calculation results of the equation of state}

The EOS shown in Eq. (14) was used to calculate the compressibility factors of pure and mixture SW fluids. Table 5 presents the calculation results of pure fluids from different models. It is observed that our model gives very good agreement with the MC computer simulation data. The results of Shen and $\mathrm{Lu}$ (1993) in a recent publication are also satisfactory while other models show relatively larger deviations. Table 6 shows a comparison of the calculated compressibility factors from various models for SW fluids at different $\lambda$ values. It is observed that the models from $\mathrm{Lu}$ and co-workers give very good agreement to the simulation data at different $\lambda$ values. Our work and other equations in Table 6 are obtained by fitting the simulation data at $\lambda$ equals to 1.5 , and yield relatively larger deviations at other $\lambda$ values than those from Lu and co-workers. In deriving the EOS of Eq. (14), we have fixed the $\lambda$ value to be 1.5 . The other parameters of this EOS are regressed by using experimental saturated properties of pure real fluids. Our EOS, Eq. (14), gives good calculated compressibility factors for pure SW fluids at $\lambda=1.5$ (as shown in Table 4), and is applied for real fluid calculations in this research where $\lambda$ is also fixed as 1.5 . Table 7 shows the compressibility factors of various binary mixtures of SW fluids calculated from different models. As has been mentioned by Guo et al. 
Table 6

A comparison of the calculated compressibility factors with Monte Carlo simulation data at various $\lambda$ values

\begin{tabular}{llllllllll}
\hline$x$ & $k T / \epsilon$ & $\rho \sigma^{3}$ & MC $^{\text {a }}$ & LC & GWL-I & GWL-II & SL & TL & This work \\
\hline 1.13 & 1.00 & 0.4 & 1.390 & 2.049 & 2.081 & 2.005 & 1.484 & 1.583 & 2.007 \\
1.13 & 1.00 & 0.6 & 2.230 & 3.416 & 3.371 & 3.470 & 2.604 & 2.226 & 3.562 \\
1.13 & 1.00 & 0.8 & 3.530 & 6.601 & 7.13 & 6.549 & 5.430 & 3.540 & 6.790 \\
1.38 & 1.50 & 0.4 & 0.970 & 1.306 & 1.489 & 1.212 & 1.172 & 0.930 & 1.221 \\
1.38 & 1.50 & 0.6 & 1.680 & 2.018 & 2.896 & 2.158 & 2.178 & 1.458 & 2.327 \\
1.38 & 1.50 & 0.8 & 4.000 & 4.776 & 6.087 & 4.594 & 4.890 & 3.941 & 5.140 \\
1.63 & 2.00 & 0.4 & 0.720 & 0.629 & 0.984 & 0.495 & 0.800 & 0.678 & 0.510 \\
1.63 & 2.00 & 0.6 & 1.660 & 0.726 & 2.154 & 0.945 & 1.642 & 1.673 & 1.172 \\
1.63 & 2.00 & 0.8 & 5.380 & 3.097 & 5.122 & 2.773 & 4.188 & 5.181 & 3.597 \\
2.000 & 6.00 & 0.4 & 1.480 & 1.160 & 1.505 & 1.083 & 1.427 & 1.472 & 1.085 \\
2.000 & 6.00 & 0.6 & 2.820 & 1.676 & 2.800 & 1.835 & 2.626 & 2.762 & 1.968 \\
2.000 & 6.00 & 0.8 & 5.500 & 4.359 & 5.819 & 4.063 & 5.527 & 5.498 & 4.654 \\
AAD\% & & & & 38.0 & 39.7 & 38.7 & 16.3 & 3.8 & 37.8 \\
\hline
\end{tabular}

${ }^{\mathrm{a}} \mathrm{MC}$ computer simulation data are taken from Henderson et al. (1980).

(1990b), the EOS for SW fluid mixtures cannot be derived from either the LC or GWL-II coordination number model, owing to the complex temperature summation terms in their models. Only the EOS derived from LS and GWL-I coordination number models are used for comparison in Table 7. It is again demonstrated that our EOS gives better results than those from the LS or GWL-I coordination number models. Graphical comparisons are presented in Fig. 4 for pure fluids where the results from our EOS have superior agreement with MC computer simulation data than that from other equations. Fig. 5 presents the mixture compressibility factors calculated from three models. It is shown that all models have good results at low densities. Our model, however, has better agreement with the MC computer simulated data at higher density regions.

The EOS, Eq. (14), was employed in real fluid calculations. For each pure fluid, we need a molecular size parameter, $\sigma$, and an energy parameter, $\epsilon / \mathrm{k}$. The energy parameter is written in a temperature-dependent form

$$
\epsilon / k=(\epsilon / k)_{0}\left[1+m\left(1-T_{\mathrm{r}}^{0.5}\right)\right]^{2}
$$

Table 7

The comparison of various models in the calculation of the compressibility factors of SW fluid mixtures at $\lambda=1.5$

\begin{tabular}{lllll}
\hline Case & $\begin{array}{l}\text { No. of } \\
\text { Data pts. }\end{array}$ & \multicolumn{2}{l}{ Absolute average deviation in $Z^{\text {a }}$} \\
\cline { 3 - 5 } & & LS & GWL-I & This work \\
\hline I & 48 & 0.49 & 0.67 & 0.22 \\
II & 44 & 0.55 & 0.73 & 0.25 \\
I11 & 48 & 0.61 & 0.79 & 0.27 \\
IV & 16 & 0.52 & 0.68 & 0.23 \\
Grand average & & 0.55 & 0.73 & 0.24 \\
\hline
\end{tabular}

Case I-IV are taken from the same data sources as Case I-IV in Table 2.

${ }^{\mathrm{a}} \mathrm{AAD}=(1 / N) \sum_{i}\left(a b s\left(Z_{\mathrm{i}, \mathrm{MC}}-Z_{\mathrm{i}, \mathrm{cal}}\right), N\right.$ : total number of data points. 


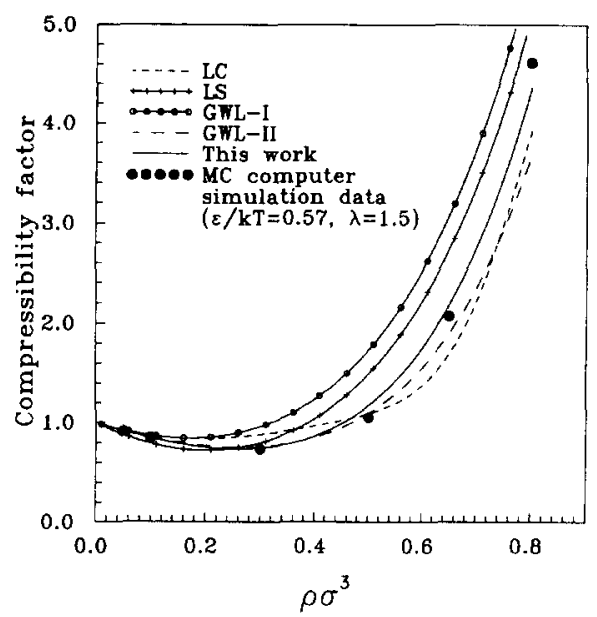

Fig. 4. Comparison of the calculated compressibility factors of pure SW fluids by various models (MC computer simulation data: Guo et al., 1990a).

The three parameters, $\sigma,(\epsilon / k)_{0}$ and $m$, for each pure fluid are evaluated by fitting their saturated properties. The following objective function is minimized in this work

$$
O . F .=\Sigma\left(\left|\frac{P^{\mathrm{vp}, \mathrm{cal}}-P^{\mathrm{vp}, \exp }}{P^{\mathrm{vp}, \exp }}\right|+\left|\frac{V^{\mathrm{L}, \mathrm{cal}}-V^{\mathrm{L}, \exp }}{V^{\mathrm{L}, \exp }}\right|+\left|\frac{V^{\mathrm{V}, \mathrm{cal}}-V^{\mathrm{V}, \exp }}{V^{\mathrm{V}, \exp }}\right|\right)^{2}
$$

where the summation is over all data points on the saturation curve. Table 8 lists the pure component parameters for $n$-paraffins and the calculated results of pure component properties. Generally, this EOS yields satisfactory results on vapor pressures and saturated vapor and liquid volumes. We have compared our calculation results with those computed from a theoretically-based SAFT EOS (Huang



Fig. 5. Comparison of the calculated compressibility factors of $\mathrm{SW}$ fluid mixture by various models $\left(\epsilon_{11} / k T=0.5\right.$, $\epsilon_{22} / k T=1.0, \epsilon_{12} / k T=0.707, x_{1}=0.796$ and $\sigma_{22} / \sigma_{11}=1$; (MC computer simulation data: Lee and Chao, 1987). 
Table 8

Comparison of the calculated results of pure n-paraffins by using the SAFT EoS and the EoS developed in this work

\begin{tabular}{|c|c|c|c|c|c|c|c|c|c|c|c|c|}
\hline \multirow[t]{2}{*}{ Compound } & \multirow{2}{*}{$\begin{array}{l}\text { Temp. } \\
\text { range } \\
(\mathrm{K})\end{array}$} & \multirow{2}{*}{$\begin{array}{l}\text { No. } \\
\text { of } \\
\text { Data } \\
\text { pts }\end{array}$} & \multirow[t]{2}{*}{$(\epsilon / k)_{0}$} & \multirow[t]{2}{*}{$\sigma$} & \multirow[t]{2}{*}{$m$} & \multicolumn{2}{|c|}{$\mathrm{AAD} \%$ in $V^{\mathrm{L}}$} & \multicolumn{2}{|c|}{$\mathrm{AAD} \%$ in $V^{\mathrm{V}}$} & \multicolumn{2}{|c|}{$\mathrm{AAD} \%$ in $P^{\text {sat }}$} & \multirow{2}{*}{$\begin{array}{l}\text { data } \\
\text { sources }\end{array}$} \\
\hline & & & & & & $\overline{\text { SAFT }}$ & $\begin{array}{l}\text { This } \\
\text { work }\end{array}$ & $\overline{\text { SAFT }}$ & $\begin{array}{l}\text { This } \\
\text { work }\end{array}$ & $\overline{\text { SAFT }}$ & $\begin{array}{l}\text { This } \\
\text { work }\end{array}$ & \\
\hline$\overline{\text { methane }}$ & $95-183$ & 40 & 152.19 & 3.48 & -0.032 & 0.20 & 1.02 & 0.62 & 2.60 & 0.27 & 1.39 & 1 \\
\hline ethane & $168-295$ & 27 & 248.64 & 3.96 & 0.063 & 1.25 & 0.73 & 4.34 & 2.91 & 1.92 & 1.50 & 1 \\
\hline propane & $205-357$ & 25 & 295.64 & 4.39 & 0.124 & 1.89 & 0.80 & 5.26 & 1.59 & 1.89 & 2.56 & 1 \\
\hline butane & $236-392$ & 25 & 342.86 & 4.76 & 0.152 & 2.56 & 0.68 & 2.96 & 1.35 & 1.23 & 1.59 & 1 \\
\hline pentane & $262-454$ & 27 & 379.22 & 5.10 & 0.2 & 2.96 & 0.68 & 4.43 & 2.67 & 1.60 & 1.95 & 1 \\
\hline hexane & $282-485$ & 28 & 411.85 & 5.39 & 0.24 & 3.35 & 0.69 & 4.27 & 2.06 & 1.34 & 2.19 & 1 \\
\hline heptane & $304-523$ & 28 & 440.84 & 5.67 & 0.28 & 3.30 & 0.92 & 3.44 & 1.76 & 1.27 & 1.51 & 1 \\
\hline octane & $336-552$ & 28 & 462.34 & 5.92 & 0.33 & 3.57 & 0.73 & 4.34 & 1.94 & 1.40 & 2.85 & 1 \\
\hline nonane & $344-424$ & 15 & 492.09 & 6.13 & 0.34 & 1.42 & 1.34 & 0.32 & 0.21 & 0.39 & 0.30 & 1 \\
\hline decane * & $331-523$ & 35 & 516.29 & 6.3 & 0.36 & 1.67 & 3.18 & - & - & 0.58 & 0.29 & 2 \\
\hline dodecane * & $365-520$ & 30 & 551.76 & 6.73 & 0.43 & 1.69 & 2.74 & - & - & 0.31 & 0.23 & 2 \\
\hline tetradecane * & $395-559$ & 30 & 582.68 & 7.12 & 0.49 & 1.78 & 2.31 & - & - & 0.46 & 0.28 & 2 \\
\hline hexadecane & $422-594$ & 30 & 609.46 & 7.48 & 0.55 & 1.98 & 2.17 & - & - & 1.62 & 0.19 & 2 \\
\hline eicosane * & $469-652$ & 30 & 655.25 & 8.09 & 0.65 & 2.35 & 2.97 & - & - & 0.52 & 0.34 & 2 \\
\hline \multicolumn{2}{|l|}{ Grand average } & 398 & & & & 2.16 & 1.21 & 3.33 & 2.02 & 1.03 & 1.21 & \\
\hline
\end{tabular}

-: No experimental data available, ${ }^{*}$ : Saturated liquid molar volume at 1 atm are used in this comparison.

Data sources: 1. Smith and Srivastava (1986); 2. Rossini et al. (1952).

$\operatorname{AAD}(\%)=(100 / N) \sum_{i}\left(a b s\left(\frac{X_{\mathrm{i}, \exp }-X_{\mathrm{i}, \text { cal }}}{X_{\mathrm{i}, \exp }}\right)\right), N$ : total number of data points, $X: V^{\mathrm{L}}, V^{\mathrm{v}}$ or $P^{\text {sat }}$.

and Radosz, 1990). In this comparison, we have re-fitted the SAFT EOS parameters using the same experimental data sources shown in Table 8 . From the comparison shown in Table 8 , it is observed that our EOS has comparably good results in vapor pressures as those from the SAFT EOS. It also shows that our EOS has better results in calculating the saturated vapor and liquid molar volumes than

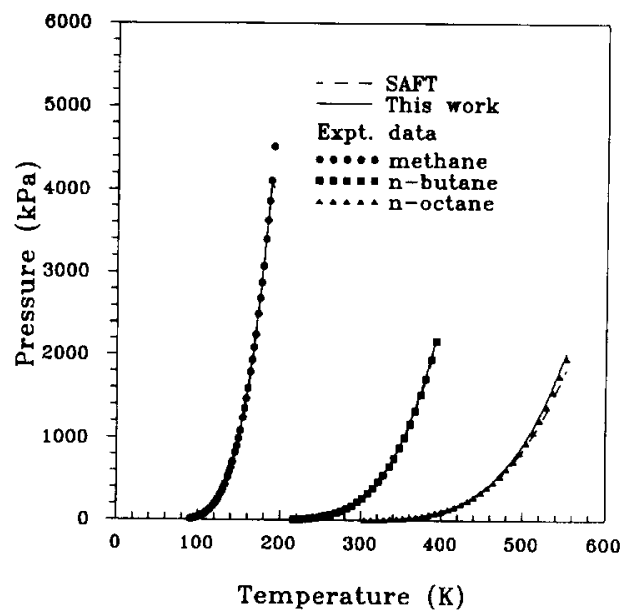

Fig. 6. Comparison of vapor pressures calculated by the SAFT EOS and this work for selected n-paraffins. 


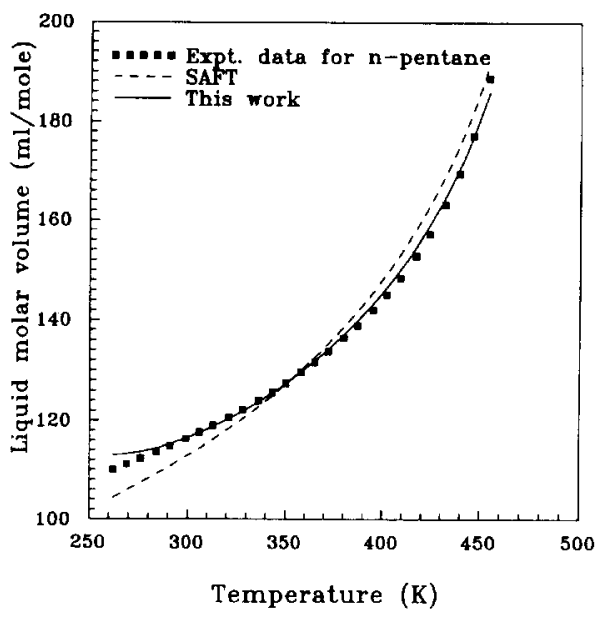

Fig. 7. Comparison of liquid molar volumes of $n$-pentane calculated by the SAFT EOS and this work.

those from the SAFT EOS. Graphical comparisons are presented in Figs. 6 and 7 for vapor pressures and liquid molar volumes, respectively. It is also noticed that the pure component parameters in our proposed EOS have regular correlations of their characteristic properties. Fig. 8 shows that the plot of $(\epsilon / k)_{0}$ against the critical temperatures of the n-paraffins has a constant slope of 0.822 . This value is in agreement with the critical energy parameter determined from other coordination number models or molecular dynamics simulation data (as shown by Guo et al., 1990b) that $\left(\epsilon / k T_{\mathrm{c}}\right.$ ) is between 0.775 and 0.84 for a pure SW fluid. Fig. 9 shows that the plot of the effective hard core volumes of n-paraffins (which is equal to $\pi \sigma^{3} / 6$ ) against the van der Waals volumes also has a constant slope of 18.2. This constant indicates that $\sigma$ has good physical significance as a volume parameter of our proposed EOS. The EOS parameter $\mathrm{m}$ also shows a nearly straight relationship with the acentric factors for n-paraffins, as indicated in Fig. 10. These relations suggest that the EOS parameters

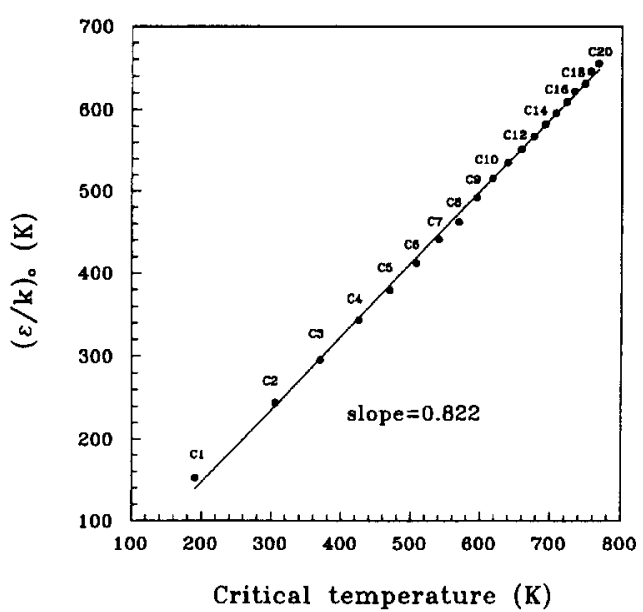

Fig. 8. Plot of $(\epsilon / k)_{0}$ against the critical temperatures of $n$-paraffins. 


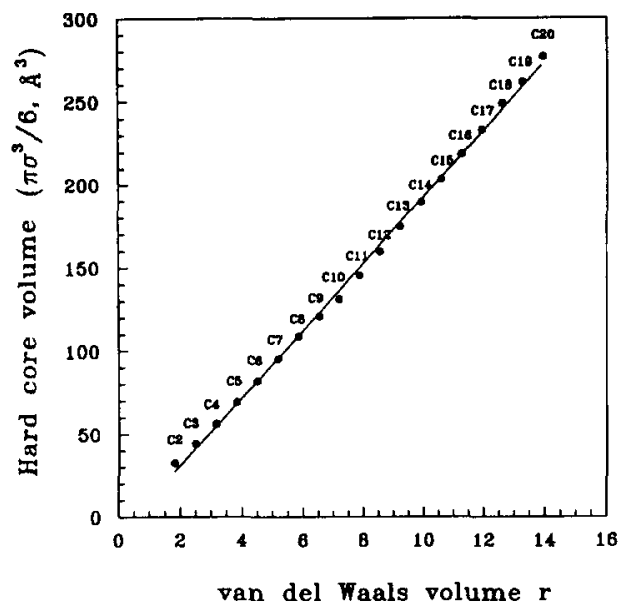

Fig. 9. Plot of the hard core volumes against the van der Waals volumes of $n$-paraffins.

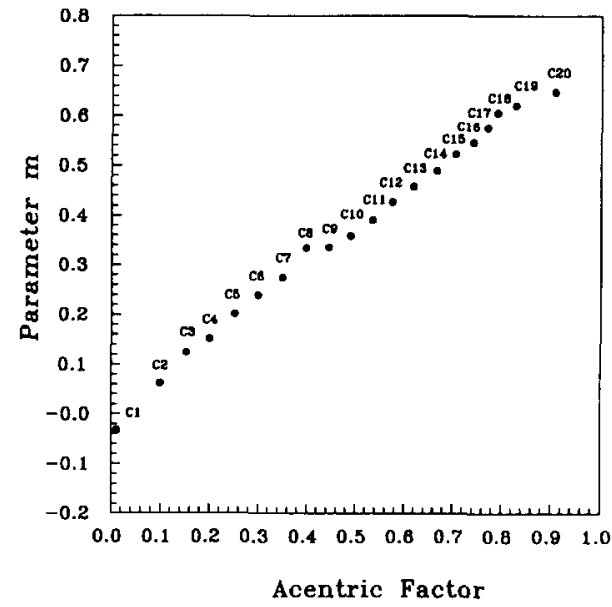

Fig. 10. Plot of the parameter $m$ against the acentric factors of $n$-paraffins.

regressed in this study have clear theoretical bases. Generalized correlations of these three EOS parameters and the extension of this EOS to fluid mixture calculations are currently undergoing where more nonpolar and polar fluids are included.

\section{Conclusion}

A new coordination number model is presented for square-well fluids. The new model satisfies the theoretical requirements at the low and high density limiting conditions. It also yields satisfactory agreement with the coordination numbers and compressibility factors of pure and mixture SW fluids at various reduced densities and energy parameters. An equation of state is derived using the generalized van der Waals partition function and the new coordination number model. Pure fluid 
parameters of this equation of state are determined for n-paraffins. Satisfactory results are presented for these real fluids and the pure fluid parameters have reasonable physical significance.

\section{List of symbols}

$f \quad$ fugacity

$g(r)$ pair distribution function

$k \quad$ Boltzmann's constant

$m$ temperature-dependent parameter in Eq. (16)

$N \quad$ number of molecules

$N_{\mathrm{c}} \quad$ total coordination number

$N_{\mathrm{ij}} \quad$ coordination number of molecule $\mathrm{i}$ around central molecule $\mathrm{j}$

$P \quad$ pressure

$Q \quad$ canonical partition function

$q \quad$ single particle partition function

$r \quad$ distance

$T$ absolute temperature

$T_{\mathrm{r}} \quad$ reduced temperature

$T^{*} \quad$ dimensionless parameter of $k T / \epsilon$

$V \quad$ volume

$V_{\mathrm{f}} \quad$ free volume

$x \quad$ mole fraction

Z compressibility factor

Greek letters

$\epsilon \quad$ depth parameter of the SW potential

$\Gamma \quad$ potential function

$\Phi \quad$ mean potential

$\eta \quad$ dimensionless parameter of $\left(\pi \rho_{\mathrm{m}} / 6\right) \sum x_{\mathrm{i}} \sigma_{\mathrm{i}}^{3}$

$\Lambda$ de Broglie wavelength

$\lambda \quad$ width parameter of the SW potential

$\rho \quad$ number density

$\rho^{*} \quad$ reduced density, $\rho \sigma^{3}$

$\sigma \quad$ molecular diameter

$\Psi \quad$ correction term for the coordination number model

$\Omega$ dimensionless parameter in the coordination number models

\section{Subscripts}

cal calculated properties

e electronic contributions

exp experimental data

i component $i$

$\mathrm{j}$ component $\mathrm{j}$ 


\section{Monte Carlo computer simulation data}

$\mathrm{m} \quad$ mixture properties

r rotational contributions

v vibrational contributions

\section{Superscripts}

$\begin{array}{ll}\text { L } & \text { liquid phase } \\ \text { V } & \text { vapor phase } \\ \text { sat } & \text { saturated property }\end{array}$

\section{Acknowledgements}

The authors are grateful to the National Science Council of the Republic of China for supporting this research.

\section{References}

Alder, B.J., Young, D.A. and Mark, M.A., 1972. Studies in molecular dynamics. X. Corrections to the augmented van der Waals theory for the square-well fluid. J. Chem. Phys., 56: 3013-3029.

Carnahan, N.F. and Starling, K.E., 1969. Equation of state for nonattracting rigid spheres. J. Chem. Phys., 51: 635-636.

Guo, M.-X., Wang, W.-C. and Lu, H.-Z., 1990a. Equations of state for pure and mixture square-well fluids. I. Coordination number models. Fluid Phase Equilibria, 60: 37-45.

Guo, M.-X., Wang, W.-C. and Lu, H.-Z., 1990b. Equations of state for pure and mixture square-well fluids. II. Equations of state. Fluid Phase Equilibria, 60: 221-237.

Henderson, D., 1976. Monte Carlo and hypernetted chain equation of state for the square-well fluid. J. Chem. Phys., 64: 5026-5034.

Henderson, D., Scalise, O.H. and Smith, W.R., 1980. Monte Carlo calculations of the equation of state of the square-well fluid as a function of well width. J. Chem. Phys., 72: 2431-2438.

Hu, Y., Ludecke, D. and Prausnitz, J.M., 1984. Molecular thermodynamics of fluid mixtures containing molecules differing in size and potential energy. Fluid Phase Equilibria, 17: 217-241.

Huang, S.H. and Radosz, M., 1990. Equation of state for small, large, polydisperse, and associating molecules. Ind. Eng. Chem. Res., 29: 2284-2294.

Lee, K.-H., Lombardo, M. and Sandler, S.I., 1985. The generalized van der Waals partition function. II. Application to the square-well fluid. Fluid Phase Equilibria, 21: 177-196.

Lee, K.-H., Sandler, S.I. and Patel, N.C., 1986. The generalized van der Waals partition function. III. Local composition models for a mixture of equal size square-well fluid. Fluid Phase Equilibria, 25: 31-49.

Lee, K.-H. and Sandler, S.I., 1987. The generalized van der Waals partition function. IV. Local composition models for mixtures of unequal-size molecules. Fluid Phase Equilibria, 34: 113-147.

Lee, R.J. and Chao, K.C., 1987. Coordination number and thermodynamics of square-well fluid mixtures. Mol. Phys. 61: $1431-1442$.

Reed, T.M. and Gubbins, K.E., 1973. Applied statistical mechanics, McGraw-Hill, New York.

Rossini, F.D., Pitzer, K.S., Amett, R.L., Braun, R.M. and Pimental, G.C., 1952. Selected values of properties of hydrocarbons and related compounds. API Project 44, Canegie, Pittsburgh.

Sandler, S.I., 1985. The generalized van der Waals partition function. I. Basic theory. Fluid Phase Equilibria, $19: 233-257$.

Shen, S. and Lu, B.C.-Y., 1993. A simple perturbed equation of state from a pseudopotential function. Fluid Phase Equilibria, 84: 9-22.

Smith, B.D. and Srivastava, R., 1986. Thermodynamic data for pure compounds. Elsevier, Amsterdam.

Tang, Y. and Lu, B.C.-Y., 1994. An analytical analysis of the square-well fluid behavior. J. Chem. Phys., $100: 6665-6671$. 